\title{
Tigecycline and homoharringtonine synergistically target myeloid leukemia cells by inhibiting mitochondrial translation through mTOR/4EBP1 pathway
}

\section{Haiyang Yang}

Zhejiang University School of Medicine First Affiliated Hospital

\section{Chen Mei}

Zhejiang University School of Medicine First Affiliated Hospital

\section{Li Ye}

Zhejiang University School of Medicine First Affiliated Hospital

\section{Yanling Ren}

Zhejiang University School of Medicine First Affiliated Hospital

\section{Hua Zhang}

Zhejiang University School of Medicine First Affiliated Hospital

\section{Kongfei Li}

Department of Hematology, Yin Zhou People Hospital

\section{Jiansong Huang}

Zhejiang University School of Medicine First Affiliated Hospital

\section{Xin Huang}

Zhejiang University School of Medicine First Affiliated Hospital

\section{Weilai Xu}

Zhejiang University School of Medicine First Affiliated Hospital

\section{Xinping Zhou}

Zhejiang University School of Medicine First Affiliated Hospital

\section{Gaixiang Xu}

Zhejiang University School of Medicine First Affiliated Hospital

\section{Lu Wang}

Zhejiang University School of Medicine First Affiliated Hospital

\section{Chuying Shen}

Zhejiang University School of Medicine First Affiliated Hospital Hongyan Tong ( $\nabla$ tonghongyan@zju.edu.cn )

Zhejiang University School of Medicine First Affiliated Hospital https://orcid.org/0000-0001-5603-4160 
Research

Keywords: Tigecycline, Homoharringtonine, AKT, mTOR, 4EBP1, myeloid leukemia

Posted Date: August 4th, 2020

DOI: https://doi.org/10.21203/rs.3.rs-42286/v1

License: (c) (i) This work is licensed under a Creative Commons Attribution 4.0 International License. Read Full License 
Tigecycline and homoharringtonine synergistically target myeloid leukemia cells by inhibiting mitochondrial translation through mTOR/4EBP1 pathway

Haiyang Yang1,2, Chen Meiı,Li YE1, Yanling Ren1, Hua Zhang1,Kongfei Li3,Jiansong Huang1,2,Xin

Huang1,2,Weilai Xu1,Xinping Zhou1,Gaixiang Xu1, Lu Wang1, Chuying Shen1,Hongyan Tong1,2*

\begin{tabular}{|l|l|}
\hline Author & Email address \\
\hline Haiyang Yang & haiyanglucky@163.com \\
\hline Chen Mei & meichenblood@yeah.net \\
\hline Li YE & yelikybs@126.com \\
\hline Yanling Ren & yanlingrenxm @163.com \\
\hline Hua Zhang & sapphirezh@126.com \\
\hline Kongfei Li & konfeelee@126.com \\
\hline Jiansong Huang & hjiansong1234@zju.edu.cn \\
\hline Xin Huang & hx0628@yahoo.com \\
\hline Weilai Xu & lpxwl2005@163.com \\
\hline Xinping Zhou & zxp1207@163.com \\
\hline Gaixiang Xu & 1505136@zju.edu.cn \\
\hline Chuying Shen & 21818095@zju.edu.cn \\
\hline Lu Wang & 11818213@zju.edu.cn \\
\hline Hongyan Tong & tonghongyan@zju.edu.cn \\
\hline
\end{tabular}

Abstract

Background: Tigecycline (TIG) is a tetracycline derivative antibiotic. Successive studies have shown that

TIG is efficacious for the treatment of some solid tumors and hematological malignant diseases both in vivo and in vitro, and drug combinations appear to provide better inhibition. To explore new drug combinations for myeloid leukemia, we compared the differential combination efficacy of TIG with several anti-leukemia drugs, and explored the mechanisms of the combination of TIG and homoharringtonine (HHT) in myeloid

11 leukemia cells both in vitro and in vivo.

Methods: Cell proliferation was assessed using MTS (3-(4,5-dimethylthiazol-2-yl)-5-(3-carboxymethoxyph- 
assays. Apoptosis was detected by flow cytometry. The combination of effects was confirmed in myeloid

15 leukemia cells and tumor-bearing mouse model. The regulation of the AKT/mTOR (mammalian target of 16 rapamycin)/4EBP1 (eukaryotic translation initiation factor 4E binding protein 1) pathway was assessed using 17 a Western blot and immunohistochemistry.

18 Results: The combination of TIG and HHT had a strong synergistic effect in myeloid leukemia cells. The use 19 of the drug combination in vivo also effectively delayed myeloid tumor development in mice. The synergistic 20 effect of this drug combination is likely to be achieved by inhibiting mitochondrial translation and down21 regulating the AKT/mTOR/4EBP1 signaling pathway.

22 Conclusion: The combination of TIG and HHT can synergistically enhance an anti-leukemia effect through 23 downregulating anti-apoptotic proteins. Inhibiting mitochondrial translation through the AKT/mTOR/4EBP1 24 pathway might be an important mechanism.

25 Keywords: Tigecycline; Homoharringtonine; AKT; mTOR; 4EBP1; myeloid leukemia

\section{Background}

Tigecycline (TIG) is a new generation of tetracycline antibiotic which contains the $\mathrm{N}, \mathrm{N}$ dimethylglycylamido substitution at position 9 of minocycline [1]. The mechanism of action is similar to that of ordinary tetracyclines, and it binds to the A site of the $30 \mathrm{~s}$ subunit of the bacterial ribosome. Moreover, 30 TIG also binds to the remaining part of the $\mathrm{H} 34$ ribosome, resulting in a stronger inhibition of bacterial 31 translation [2]. Ribosome protection and drug efflux are the primary mechanisms by which most bacteria 32 develop tetracycline resistance [3]. The steric hindrance of TIG is due to a large substituent at position 9 33 appears to surmount these resistance mechanisms [2]. 
It has been reported that TIG has an anti-tumor effect in many solid tumors and blood related diseases [4-

35 7]. The inhibition of mitochondrial translation is functionally important for the selective toxicity of TIG on

36 leukemia cells in xenograft models of human leukemia [5]. Moreover, FDA(Food and Drug Administration)-

37 approved drug screening has determined that TIG can effectively suppress $\mathrm{Rb} / \mathrm{p} 53$-deficient tumor cell

38 proliferation (e.g., triple-negative breast cancer) by inhibiting the expression of mitochondrial protein 39 translation (MPT) [4].

TIG has been shown to exhibit potential value in the clinical treatment of blood-related diseases (e.g.,

41 lymphoma and leukemia), especially in combination with chemotherapy drugs. The combination of TIG with 42 daunorubicin and cytarabine has shown a strong anti-leukemic activity both in vivo and in vitro [5]. As 43 previously reported, the mTOR/4EBP1 pathway plays an important role in regulating mRNA translation [810]. In our study, we screened and compared the combined effects and differences of TIG with Chidamide (CHI), Idarubicin (IDA), and HHT, respectively. It was found that TIG combined with HHT showed a strong synergistic effect. Further research revealed that this synergy is likely to be achieved by inhibiting mitochondrial translation and acting on the cellular mTOR/4EBP1 pathway.

Materials and methods

\subsection{Antibodies and reagents}

Antibodies specific to $\beta$-actin (\#8457), Caspase3 (\#9664), PARP(poly ADP-ribose polymerase) (\#9532),

p-Akt (\#4060), p-mTOR (\#5536), p-Raptor (\#9111), p-4EBP1 (\#13443), COX(Cyclooxygenase)-1 (\#9947) including COX-4 (\#4844), p-Rictor (\#3806), Ki-67 (\#9449) were purchased from CST (Danvers, MA). HHT was obtained from MedChemExpress (Monmouth Junction, NJ). CHI was purchased by Chipscreen Biosciences (Shenzhen, China) and IDA was purchased from the Hangzhou Minsheng Institute of 
56 Pharmaceutical Research (Hangzhou, China). TIG was gifted by the Pfizer Biopharma (Hangzhou) Co., Ltd 57 (Hangzhou, China).

\subsection{Cell lines and primary patient cells}

The myeloid leukemia cell lines included SKM-1, MOLM-13, and MDS-L cells. SKM-1 and MOLM-13 were purchased from the Cell Bank of Type Culture Collection of Chinese Academy of Science (Shanghai, China). MDS-L was presented by Professor Huang Gang (The Cincinnati Children's Hospital Medical Center). Genomic DNA microsatellites were used to identify the authenticity of these cell lines by Shanghai Biowing Applied Biotechnology (Shanghai, China). The SKM-1 and MDS-L cell lines were cultured in RPMI1640 (Corning) medium supplemented with $10 \%$ fetal bovine serum (FBS, Gibco) in a humidity cell incubator that was maintained at $37^{\circ} \mathrm{C}$ and $5 \% \mathrm{CO}_{2}$. The MOLM-13 cell lines and primary myeloid leukemia cells were cultured in Iscove's Modified Dubecco's Medium (IMDM, Corning) supplemented with 10\% FBS. The bone marrow mononuclear cells of patients were isolated by Ficoll Hypaque density gradient centrifugation and treated with erythrocyte lysate. All patients signed an informed consent form.

\subsection{Cell viability assay}

A density of either $1 \times 105$ for cell lines or $2 \times 105$ for primary patient cells were seeded into 24 -well plates. $20 \mu \mathrm{L}$ MTS solution (Promega, Madison, WI) were added to each well and incubated at $37^{\circ} \mathrm{C}$ or $4 \mathrm{~h}$. The optical delnsity (OD) values of these wells were measured using a microplate reader (Varioskan Flash, Thermo scientific) at a wavelength of $490 \mathrm{~nm}$. All cell lines and primary patient cells were tested with at least three independent replicates.

\subsection{Flow cytometry analysis}

After drug treatment, the cells were washed twice with phosphate buffer saline (PBS), and $1-5 \times 105$ cells were collected. The supernatant was centrifuged at $1500 \mathrm{rpm}$ for $5 \mathrm{~min}$, and $500 \mu \mathrm{L}$ Binding Buffer was added 
to prepare the cell suspension. Next, $6 \mathrm{~mL}$ Annexin-V FITC and $10 \mu \mathrm{L}$ PI (propidium iodide) were added, 80 incubated in dark for $5 \mathrm{~min}$, and annexin-v FITC (radiation wavelength $=488 \mathrm{~nm}$; absorption wavelength $=$ $81530 \mathrm{~nm}$ ) was detected by the FITC signal (FL1 channel). The PE signal was detected for PI staining (FL2 channel). Both signals were analyzed using a FACScan flow cytometer (Becton Dickinson, San Diego, CA).

\subsection{Western blot analysis}

106 cells were collected in each group. After suspending the cells in $40 \mu \mathrm{L}$ of RIPA

(Radioimmunoprecipitation assay ) buffer (Thermo Fisher Scientific, Waltham, MA), they were placed on ice

for $30 \mathrm{~min}$ at $4^{\circ} \mathrm{C}$, centrifugated at $12000 \times \mathrm{g}$ for $20 \mathrm{~min}$, and the protein supernatant was collected. The protein concentration in the supernatant was determined with BCA (Bicinchoninic acid) reagent (BBI life sciences, Shanghai, China) and then mixed with $4 \times$ loading buffer at $100^{\circ} \mathrm{C}$ for thermal denaturation. The protein samples were separated with SDS-PAGE (Sodium dodecyl sulfate polyacrylamide gel electrophoresis) gel (Thermo Fisher Scientific, Waltham, MA) and transferred to PVDF (Polyvinylidene fluoride) membranes (Millipore, Burlington, MA). The membrane was sealed with tris-buffered saline (TBS) containing 5\% skim milk for $1 \mathrm{~h}$ and incubated with a primary antibody on a shaker overnight at $4^{\circ} \mathrm{C}$. After washing three times with tris-buffered saline tween (TBST) buffer, the membranes were incubated with the secondary antibodies (CST, Danvers, MA) for $1 \mathrm{~h}$. The ECL reagent (Thermo Fisher Scientific, Waltham, MA) was catalyzed by an HRP (Horseradish peroxidase) reaction on the HRP-labeled secondary antibody to display the target protein bands, and the ChemiDoc MP imaging system (Bio-Rad, Hercules, CA) was used for imaging.

\subsection{Immunohistochemistry}

The stripped tumor tissue was cut into appropriately sized tissue pieces and fixed in $4 \%$ paraformaldehyde

for two days. The tissue pieces were removed from the fixing solution, rinsed with PBS for $1 \mathrm{~h}$, and proceeded. 
continuous 5- $\mu \mathrm{m}$ thick sections, dewaxed with xylene, and then the sections were fully rehydrated with

103 gradient alcohol and water. After the antigen was repaired, an appropriate amount of $3 \% \mathrm{H}_{2} \mathrm{O}_{2}$ was added

104 dropwise. The tissue was covered with $\mathrm{H}_{2} \mathrm{O}_{2}$ and incubated at room temperature for 10 min to block the endogenous peroxidase activity. The tissue was washed with PBS on a shaker three times for 5 min. Then, 5\% goat serum was prepared with PBS added dropwise, and the tissue was blocked for $1 \mathrm{~h}$. The slides were dried,

the residual liquid was aspirated, placed in a wet box, the primary antibody liquid was dropped to completely cover the tissue, and incubated overnight at $4{ }^{\circ} \mathrm{C}$ in a refrigerator. The tissues were washed three times with

PBS on a shaker at room temperature for 10 min each time. Then $5 \%$ goat serum was diluted with biotin-

labeled secondary antibody to cover the surface of the tissue and incubated at room temperature for 45 min,

before washing with PBS three times for $10 \mathrm{~min}$. An appropriate amount of avidin-labeled horseradish peroxidase was added to the tissue surface and incubated at room temperature for $30 \mathrm{~min}$. The tissue was washed three times, 5 min each time and diaminobezidin (DAB) color solution was added to the surface of the tissue and the staining was observed under a microscope. When the color was obviously brown and the background was not visible, then placed in water to stop the color reaction. The tissues were stained with hematoxylin for $1-2$ min and rinsed with water to stop the staining. The tissues were observed and pictures were obtained under the microscope after mounting. On sections that had been dewaxed and rehydrated, an appropriate amount of hematoxylin was added to cover the tissue surface, the nucleus was stained for 5 min, and the remaining staining solution was rinsed away with tap water. The tissues were quickly differentiated with $1 \%$ hydrochloric acid alcohol, rinsed with tap water, and an appropriate amount of eosin was added. The solution was stained on the surface of the tissue for $30 \mathrm{~s}$ and then rinsed with tap water. After dehydration to transparency, the tissue was observed under the microscope and photographed after sealing.

\subsection{Mouse models}


Animal experiments in this study were performed in accordance with the "Guidelines for the Care and Use

of Laboratory Animals" (NIH Publication 86-23, revised in 1985) issued by the National Institutes of Health.

The animal experiment protocol was approved by the Animal Protection and Facilities Committee of Zhejiang

University. Male nude mice of six-week-old were purchased from Zhejiang Academy of Medical Sciences.

The SKM-1 myeloid leukemia cell line $(1 \times 107$ cells per animal $)$ was subcutaneously injected into the right

forelimb flank of the mice. Seven days after cell injection, when the tumors were palpable, animals with approximately the same tumor volume were randomly divided into four groups consisting of five animals per group. The use of TIG $(50 \mathrm{mg} / \mathrm{kg} / \mathrm{d})$, HHT $(0.5 \mathrm{ng} / \mathrm{kg} / \mathrm{d})$ or a combination for two consecutive weeks. For the control group, PBS was used for the injection. Both the drugs and PBS were intraperitoneally injected. The mice were weighed every day and calipers were used to calculate the tumor volume every two days using the formula: $\pi / 6$ length $\times$ width2. Two weeks later, in accordance with ethical animal practices, each group of mice was killed, and the tumors were collected for further testing.

\subsection{Statistical analysis}

The significant differences among the groups was determined using a one-way ANOVA (Analysis of Variance) followed by a post-hoc Bonferroni's multiple comparison test. For non-parametric data, a KruskalWallis followed by a Dunn's multiple comparison test was used. The minimal level of significance was $\mathrm{p}<$ 0.05. All data were presented as the mean \pm standard deviation (SD). GraphPad PrismV 5.0 (GraphPad Software, San Diego, CA) was used for statistical analysis. * $\mathrm{p}<0.05 ; * * \mathrm{p}<0.01 ; * * \mathrm{p}<0.001 ; * * * *<$ 0.0001 .

\section{Results}

\section{TIG-HHT combination can synergistically inhibit the growth of myeloid leukemia cells}

To investigate the effect of the drug combination on the proliferation of myeloid leukemia cells, we used 
maximal inhibitory concentration) of SKM-1 at $72 \mathrm{~h}$ was calculated to determine the appropriate concentration

of each drug (Table S1). In the experiment, SKM-1 was exposed to $72 \mathrm{~h}$ in TIG, HHT, CHI, and IDA. In all

combination groups of SKM-1, the effect of the drug combination on the cells was evaluated at 24,48 , and

72 h. Next, we used the median-effect method to calculate the combination index of each group to evaluate

the combined effect of each group using CalcuSyn software[11]. When TIG was combined with CHI and IDA, most dose combinations had CI (Combination Index) values above 1 at 24,48 , and $72 \mathrm{~h}$ after the drug combination. Although the duration of action was prolonged, they did not show a better combined effect.

When TIG and HHT were administered at the same time, the CI value of almost all metering combinations was less than 1 , and the CI value of each metering combination of $72 \mathrm{~h}$ was less than $24 \mathrm{~h}$ and $48 \mathrm{~h}$ (Figure 1). This indicates that the combination of TIG-HHT shows a strong anti-leukemia response. The synergistic and cumulative effects were evaluated over time. At least three independent experiments were performed with three replicates in each group.

To confirm the synergistic effect of the TIG-HHT combination on different myeloid leukemia cells, we measured the IC50 of TIG and HHT for $72 \mathrm{~h}$ in MOLM-13, MDS-L, and the primary patient cells (Table S2).

Based on the IC50 of each drug, we measured MOLM-13, MDS-L, and patient primary cells for $72 \mathrm{~h}$. The effects of different concentrations of TIG in combination with HHT on the viability of the cells was measured by an MTS assay after a $72 \mathrm{~h}$ incubation. The data were analyzed with Calcusyn software to generate a CI versus fractional effect (cell death) plot showing the effect of the combination of TIG with HHT. After $72 \mathrm{~h}$ of drug treatment, the CI values of almost all metered combinations were less than 1, indicating that the TIGHHT combination showed a strong synergistic inhibitory effect in both primary and myeloid leukemia cells. 
To test the effect of the TIG-HHT drug combination on the formation of myeloid leukemia cell clones in

vitro, $1 \times 103$ SKM-1, MOLM-13, and MDS-L cells were respectively inoculated on a methylcellulose semi-

solid medium. Clone formation experiments were performed and the number of clones was counted using

image-J software (Figure 3A). The drug environment of myeloid leukemia cell methylcellulose semi-solid

medium was: SKM-1 plus TIG $7.5 \mu \mathrm{g} / \mathrm{mL}$ was treated with HHT $8 \mathrm{ng} / \mathrm{mL}$; MOLM-13 plus TIG $5 \mu \mathrm{g} / \mathrm{mL}$ was

treated with $1 \mathrm{ng} / \mathrm{mL}$ HHT for $72 \mathrm{~h}$; MDS-L plus $6 \mathrm{mg} / \mathrm{mL}$ TIG was treated with $6 \mathrm{ng} / \mathrm{mL}$ HHT; and the

control group was treated with monotherapy and PBS. The use of TIG or HHT alone had a significant inhibitory effect on the colony forming ability of leukemia cells, and the addition of TIG and HHT at the same

time shows a stronger blow to cell colony formation (Figure 3A and C). Combination treatment compared with single treatment and combination treatment compared to the control groups was statistically significant ( $\mathrm{P}<0.05$ for SKM-1 and MOLM-13; $\mathrm{P}<0.01$ for MDS-L).

\section{TIG-HHT combination effectively restrains tumor growth in subcutaneous myeloid leukemia mouse}

\section{models}

To explore the effects of drug combinations in vivo, we performed combination treatments, single treatments, and PBS treatments (control) on a myeloid leukemia mouse model established by a subcutaneous injection of SKM-1 in mice. After seven days, the tumor grew to a size of about $50 \mathrm{~mm} 3$. We designed a treatment plan according to the report. Both the TIG and HHT group were injected once a day, and the Control group was injected with a corresponding PBS solution. Compared with the control group, TIG and HHT single-drug treatment both delayed tumor growth, and the combination showed higher efficacy $(\mathrm{p}<0.005$, Tukey's t-test after a one-way ANOVA) (Figure 4). Immunohistochemistry and a Western blot detection of the corresponding proteins showed that both single and combined drugs inhibited mitochondrial translation in vivo. 
To test whether the inhibitory effect of TIG and HHT on cell viability was achieved by inducing and

enhancing apoptosis, we performed Annexin V and PI staining on the cells treated with the drug, and analyzed

the proportion of apoptotic cells by flow cytometry. The untreated cell group served as a control. The myeloid leukemia cell lines, SKM-1, MOLM-13, and patient primary cells were incubated with TIG monotherapy or combination therapy for $72 \mathrm{~h}$, and apoptosis was quantified by Annexin V/PI staining. Myeloid leukemia cells were treated with different concentrations of the drugs: SKM-1 plus $7.5 \mathrm{mg} / \mathrm{mL}$ TIG were treated with 8 $\mathrm{ng} / \mathrm{mL}$ HHT for $72 \mathrm{~h}$; the control group was treated with monotherapy and PBS; and MOLM- 13 plus $5 \mathrm{mg} / \mathrm{mL}$ TIG was performed with $1 \mathrm{ng} / \mathrm{mL}$ HHT for $72 \mathrm{~h}$. As shown in Figure 3B, compared with the single-agent group and the blank group, the apoptotic rate of the cells increased significantly (Figure 3B). The mechanism of drug-induced apoptosis was studied by a Western blot analysis of the expression of cleaved caspase-3 and cleaved PARP, and the strong pro-apoptotic effect of the drug combination was revealed on a molecular level. The expression of cleaved caspase- 3 and cleaved PARP changed, and the protein mass in the two drug lanes was significantly lower than that of the single drug group and the control group (Figure 3D). To further confirm that treatment with TIG and HHT alone or in combination can also inhibit cell viability by inducing and enhancing apoptosis in vivo, we examined the expression of cleaved caspase-3 and cleaved PARP in mouse subcutaneous tumor tissue cells by immunoblotting (Figure 5A). Compared with the single drug and the blank groups, the amounts of combined histones was significantly reduced. The immunohistochemistry of the subsequent tissue sections more intuitively confirmed a strong pro-apoptotic effect of the combined group (Figure 4E).

\section{TIG-HHT combination inhibited mitochondrial translation by down-regulation of COX-1}

In studies using FDA-approved drugs to screen leukemia cell therapies, the application of yeast wholegenome screening technology confirmed that the specific inhibition of mitochondrial translation is the mechanism of TIG's anti-leukemia effect [5]. Thus, we used a Western blot to identify the DNA and proteins 
extracted from myeloid leukemia cells and tumor tissues. myeloid leukemia cells were treated with different

concentrations of drugs: SKM-1 plus $7.5 \mathrm{mg} / \mathrm{mL}$ TIG was treated with $8 \mathrm{ng} / \mathrm{mL}$ HHT for $72 \mathrm{~h}$, and the control group was treated with monotherapy and PBS. MOLM-13 plus $5 \mathrm{mg} / \mathrm{mL}$ TIG was treated with $1 \mathrm{ng} / \mathrm{mL}$ HHT for $72 \mathrm{~h}$, and MDS-L plus $6 \mathrm{mg} / \mathrm{mL}$ TIG was treated with $6 \mathrm{ng} / \mathrm{mL}$ HHT for $72 \mathrm{~h}$. Each cell line was treated with monotherapy and PBS was used as a control group. Compared with treatment of TIG or HHT alone, treatment with a combination of TIG-HHT caused a significant down-regulation of the mitochondrial gene, COX-1, expression in myeloid leukemia cells, where the nuclear gene COX-4 did not change significantly (Figure 5B). Tissue-specific protein testing revealed that the combined drug group down-regulated COX-1 gene expression in the tumor cells of subcutaneous myeloid leukemia mice without affecting the expression of the COX-4 gene (Figure 4E and 5B). SKM-1 was further exposed to a combination of drugs with different concentrations, and the inhibitory effect on the cells showed a stronger mitochondrial translation inhibitory effect as the combination drug concentration increased (Figure 5C).

\section{TIG-HHT combination synergistically inhibited the mTOR/4EBP1 pathway}

Western blot was used to identify the proteins extracted from both myeloid leukemia cells and tumor tissues. Cells were treated with different concentrations depending on the concentration of each compound used to inhibit cell proliferation: SKM-1 was exposed to $7.5 \mu \mathrm{g} / \mathrm{mL}$ TIG and $8 \mathrm{ng} / \mathrm{mL}$ HHT, and MOLM-13 was treated with $5 \mathrm{mg} / \mathrm{mL}$ TIG and $1 \mathrm{ng} / \mathrm{mL}$ HHT. MDS-L was exposed to $6 \mu \mathrm{g} / \mathrm{mL}$ TIG and $6 \mathrm{ng} / \mathrm{mL}$ HHT. Three groups of myeloid leukemia cells were treated for $72 \mathrm{~h}$, and the single drug or PBS group were used as a control. The mitochondrial translation regulators, mTOR, 4EBP1, and AKT proteins were down-regulated in combined or single drug group compared to the control group. The combined TIG-HHT significantly enhanced the inhibition of protein phosphorylation. We tested the level of phosphorylation of the constituent subunit Raptor, a component of mTOR complex 1 (mTORC1) and the constituent subunit Rictor, a component of mTOR complex 2 (mTORC2). When TIG-HHT co-administration, the p-Rictor and p-Rictor were 
significantly down-regulated, suggesting that the drug combination exerted a stronger inhibition on the

phosphorylation of mTOR pathway proteins. The results also showed that the expression of the mitochondrial gene COX-1, and the levels of phosphorylation of AKT and 4EBP1 were reduced to a uniform level when cultured with TIG-HHT combination (Figure 5B, 5D).

\section{Discussion}

Tigecycline (TIG) has been reported to affect cellular translation and tumor proliferation [5, 12]. homoharringtonine (HHT) is an effective drug for myeloid leukemia[11]. In our study, we found the combination of TIG and HHT had a strong synergistic effect in myeloid leukemia cells. The combination in vivo also effectively delayed myeloid tumor development in mice. The synergistic effect of the combination might be achieved by inhibiting mitochondrial translation and downregulating the AKT/mTOR/4EBP1 signaling pathway.

Mitochondria are the source of power for various cellular activities and biochemical reactions. TIG can specifically inhibit mitochondrial gene translation by down-regulating the expression of elongation factor, $\mathrm{Tu}$, thereby achieving cytotoxic effects on leukemia cells [5]. Moreover, HHT can also directly inhibit the mRNA translation in myeloid leukemia cells [13]. We explored the mitochondrial gene-encoded protein subunit COX-1 and the nuclear protein-encoded respiratory chain complex 4 protein subunit COX-4 to reveal the differential effects of the drug on nuclear translation and mitochondrial translation. In this study, we found HHT specifically inhibits mitochondrial translation. Moreover, both the single TIG and combination treatment showed levels of COX-1 protein but not COX-4 down-regulation significantly compared to the control group in each cell line. Further use of the TIG-HHT combination showed a stronger inhibition of mitochondrial translation as the concentration increased in the refractory leukemia cell line SKM-1 (Figure 5B). In vivo experiments also showed that the level of COX-1 gene expression in the combination group was down- 
regulated in the leukemia cells of subcutaneous tumor mice without affecting the expression of the COX-4

261 gene (Figure 4E and 5B). Both the in vivo and in vitro experiments suggested that the combination showed a stronger inhibition of mitochondrial function, which might represent an important mechanism for the synergistic effects.

The mTOR/4EBP1 pathway plays an important role in regulating mRNA translation [8-10]. mTOR can affect translation by regulating the transcription of ribosomal RNA and tRNA $[19,20]$. In addition, activated mTOR regulates translation through the phosphorylation of eukaryotic translation initiation factor $4 \mathrm{E}$ (eIF4E) binding protein 1 and an analog of the allosteric mTOR inhibitor rapamycin [21,22].There is a mutual regulation between mTOR and AKT [15-18]. mTOR complex 1 (mTORC1) is a downstream target molecule of AKT, and mTOR complex 2 (mTORC2) can target and activate phosphorylated AKT. In this study, both TIG and HHT down-regulated Rictor phosphorylation, which is an important subunit of mTORC2 in leukemia cells. A reduction in activated mTORC2 resulted in decreased AKT phosphorylation, and mTORC1 activation downstream of AKT was also reduced. The combined use of TIG and HHT further enhanced the inhibitory effect on the level of AKT/mTOR pathway phosphorylation, which may be the mechanism of the synergistic effect of the drugs.

The inhibition of eIF4E expression or the re-expression of 4EBP1 restores the sensitivity of the cell to apoptosis[22]. HHT can effectively inhibit the growth of p-eIF4E-expressing myeloid leukemia cells and primary leukemia cells by down-regulating the level of p-eIF4E and its downstream oncoprotein, Mcl-1, as well as by increasing apoptosis [20]. Our study shown TIG may enhance the pro-apoptotic properties of HHT on myeloid leukemia cells and was molecularly validated, as previously reported [23]. We hypothesized that this phenomenon is one of the mechanisms of the synergistic effects of TIG-HHT combination treatment on myeloid leukemia cells. 
It has been reported that AKT regulates mitochondrial respiratory capacity through 4EBP1[22].The reexpression of 4EBP1 can be used as a treatment for blood-related diseases that induce resistance to mTOR inhibitors due to a lack of 4EBP [22]. In our study, both the in vivo and in vitro results showed that changes in the level of intracellular 4EBP1 phosphorylation was consistent with the expression of the mitochondrial gene COX-1, which suggested that there might be a link between Akt/mTOR/4EBP1 and mitochondrial respiratory function.

\section{Conclusion}

We found that TIG combined with HHT has a synergistic anti-leukemia effect in vitro and significant effect on tumor volume reduction and apoptosis induction in myeloid cells and a xenograft mouse model. The downregulating mitochondrial translation and the AKT/mTOR/4EBP1 signaling pathway might explain this effect. These findings suggest clinical potential in administration of TIG and HHT in the treatment of myeloid 293 leukemia.

\section{Ethics approval and consent to participate}

Animal experiments in this study were performed in accordance with the "Guidelines for the Care and Use of Laboratory Animals" (NIH Publication 86-23, revised in 1985) issued by the National Institutes of Health. The animal experiment protocol was approved by the Animal Protection and Facilities Committee of Zhejiang University. All works were made to minimize the suffering of the animals.

\section{Consent for publication}

All authors of the article are happy to publish the article in the journal. 
The data and materials used to support the findings of this study are available from the corresponding author upon request.

\section{Competing interests}

305 The authors declare that they have no competing interests.

\section{$306 \quad$ Funding}

This work was supported by grants from National Natural Science Foundation of China (Grant number:81970117, 81470290, and 81800121).

\section{Authors' contributions}

HYT and HYY performed most experiments, analyzed the data, wrote the manuscript; CM, LY, YLR, HZ,

JSH, XH, WLX, LYM, XPZ, and GXX participated in the data analysis, provided animals, acquired and managed patients, provided facilities support; LW and CYS helped to correct the manuscript. HYT designed the overall study and supervised the experiments. All authors read and approved the final manuscript.

\section{Acknowledgments}

We would like to thank Professor Huang Gang for providing the MDS-L cell line, Chipscreen biosciences

(Shenzhen Weixin Biotechnology Co., Ltd.) for providing us the agent CHI, and Zhejiang Academy of 317 Medical Sciences providing us the nude mice and venues for animal experiments.

\section{Authors' information}

1Department of Hematology, the First Affiliated Hospital of Zhejiang University, Hangzhou 310003, 
Hospital, Ningbo 315040, Zhejiang Province, People’s Republic of China.

\section{REFERENCES}

Sum PE, Lee VJ, Testa RT, Hlavka JJ, Ellestad GA, Bloom JD, Gluzman Y, Tally FP: Glycylcyclines. 1. A new generation of potent antibacterial agents through modification of 9-aminotetracyclines. Journal of medicinal chemistry 1994, 37(1):184-188.

2. Zhanel GG, Homenuik K, Nichol K, Noreddin A, Vercaigne L, Embil J, Gin A, Karlowsky JA, Hoban DJ: The glycylcyclines: a comparative review with the tetracyclines. Drugs 2004, 64(1):63-88.

3. Speer BS, Shoemaker NB, Salyers AA: Bacterial resistance to tetracycline: mechanisms, transfer, and clinical significance. Clinical microbiology reviews 1992, 5(4):387-399.

4. Jones RA, Robinson TJ, Liu JC, Shrestha M, Zacksenhaus EJJoCl: RB1 deficiency in triple-negative breast cancer induces mitochondrial protein translation. 2016, 126(10):3739-3757.

5. Skrtic M, Sriskanthadevan S, Jhas B, Gebbia M, Wang X, Wang Z, Hurren R, Jitkova Y, Gronda M, Maclean N et al: Inhibition of mitochondrial translation as a therapeutic strategy for human acute myeloid leukemia. Cancer Cel/ 2011, 20(5):674-688.

6. Jia X, Gu Z, Chen W, Jiao J: Tigecycline targets nonsmall cell lung cancer through inhibition of mitochondrial function. Fundamental \& Clinical Pharmacology 2016, 30(4):297-306.

7. Yang R, Yi L, Dong Z, Ouyang Q, Zhou J, Pang Y, Wu Y, Xu L, Cui H: Tigecycline Inhibits Glioma Growth by Regulating miRNA-199b-5p-HES1-AKT Pathway. Mol Cancer Ther 2016, 15(3):421-429.

8. $\quad$ Yang Q, Guan K-L: Expanding mTOR signaling. Cell Research 2007, 17(8):666-681.

9. Mayer. C, Zhao. J, Yuan. X, Grummt. I: mTOR-dependent activation of the transcription factor TIF-IA links rRNA synthesis to nutrient availability GENES \& DEVELOPMENT 2004, 18:423-434.

10. Michels AA, Robitaille AM, Buczynski-Ruchonnet D, Hodroj W, Reina JH, Hall MN, Hernandez N: mTORC1 Directly Phosphorylates and Regulates Human MAF1. Molecular and Cellular Biology 2010, 30(15):3749-3757.

11. Chou TC, Talalay P: Quantitative analysis of dose-effect relationships: the combined effects of multiple drugs or enzyme inhibitors. Advances in enzyme regulation 1984, 22:27-55.

12. Hu H, Dong Z, Tan P, Zhang Y, Liu L, Yang L, Liu Y, Cui H: Antibiotic drug tigecycline inhibits melanoma progression and metastasis in a p21CIP1/Waf1-dependent manner. Oncotarget 2016, 7(3):3171-3185.

13. Pal I, Safari M, Jovanovic M, Bates SE, Deng C: Targeting Translation of mRNA as a Therapeutic Strategy in Cancer. Curr Hematol Malig Rep 2019, 14(4):219-227.

14. Nakagawa T, Matozaki S: The SKM-1 Leukemic Cell Line Established from a Patient with Progression to Myelomonocytic Leukemia in Myelodysplastic Syndrome (MDS)-Contribution to Better Understanding of MDS. Leukemia \& Lymphoma 1995, 17(3-4):335-339.

15. Hresko RC, Mueckler M: mTOR·RICTOR Is the Ser473Kinase for Akt/Protein Kinase B in 3T3-L1 Adipocytes. Journal of Biological Chemistry 2005, 280(49):40406-40416.

16. Sarbassov DD, Guertin DA, Ali SM, Sabatini DM: Phosphorylation and regulation of Akt/PKB by the rictormTOR complex. Science 2005, 307(5712):1098-1101.

17. Gu. Y, Zhou. H, Gan. Y, Zhang. J, Chen. J, Gan. X, Li. H, Zheng. W, Meng. Z, Ma. X et al: Small-molecule induction of phospho-elF4E sumoylation and degradation via targeting its phosphorylated serine 209 residue. Oncotarget 2015, 6:15111-15121.

18. Koritzinsky M, Goo CK, Lim HY, Ho QS, Too H-P, Clement M-V, Wong KP: PTEN/Akt Signaling Controls 
Mitochondrial Respiratory Capacity through 4E-BP1. PLOS ONE 2012, 7(9).

19. Sun Q, Li S, Li J, Fu Q, Wang Z, Li B, Liu SS, Su Z, Song J, Lu D: Homoharringtonine regulates the alternative splicing of $\mathrm{Bcl}-\mathrm{x}$ and caspase 9 through a protein phosphatase 1-dependent mechanism. BMC Complement Altern Med 2018, 18(1):164.

20. Zhou H, Xu RZ, Gu Y, Shi PF, Qian S: Targeting of phospho-elF4E by homoharringtonine eradicates a distinct subset of human acute myeloid leukemia. Leuk Lymphoma 2018:1-13.

21. Jacinto E, Facchinetti V, Liu D, Soto N, Wei S, Jung SY, Huang Q, Qin J, Su B: SIN1/MIP1 maintains rictor-mTOR complex integrity and regulates Akt phosphorylation and substrate specificity. Ce//2006, 127(1):125-137.

22. Sobol RW, Mallya S, Fitch BA, Lee JS, So L, Janes MR, Fruman DA: Resistance to mTOR Kinase Inhibitors in Lymphoma Cells Lacking 4EBP1. PLOS ONE 2014, 9(2).

23. Cao J, Feng H, Ding NN, Wu QY, Chen C, Niu MS, Chen W, Qiu TT, Zhu HH, Xu KL: Homoharringtonine combined with aclarubicin and cytarabine synergistically induces apoptosis in $t(8 ; 21)$ leukemia cells and triggers caspase-3-mediated cleavage of the MYELOID LEUKEMIA1-ETO oncoprotein. Cancer Med 2016, 5(11):3205-3213.

Figure Legends

Figure 1. TIG has in vitro antileukemia activity in combination with HHT. The combination index (CI)

of TIG used in combination with IDA, HHT, or CHI in the myeloid leukemia cell line, SKM-1, was determined by MTS staining after a three day drug culture and was calculated using CalcuSyn software (three experiments each). The effect of a $72 \mathrm{~h}$ exposure of SKM-1 cells on different concentrations of TIG in combination with IDA, HHT, and CHI on cell viability was measured using an MTS assay after a $72 \mathrm{~h}$ incubation. The data were analyzed using Calcusyn software to generate a CI versus fractional effect (cell death) plot showing the effect of a combination of TIG with IDA, HHT, or CHI. CI $<1$ indicates synergism.

Figure 2. Combination treatment using compounds targeting leukemic cells and patient primary cells identifies a synergistic effect. A. CI plots of DAC and IDA sequential combination in human leukemia cell lines. Cells were treated as previously described. The combinations were used at fixed molar ratios based on the IC50 values of each drug. The effects of the combinations were estimated using the CalcuSyn software, which was developed based on the median-effect method. $\mathrm{CI}<0.8$ indicates synergy; $\mathrm{CI}=0.8$ to 1.2 is additive; 
and CI > 1.2 indicates antagonism. B. The effect of a $72 \mathrm{~h}$ exposure on SKM-1, MDS-L, and MOLM-13 cells

391 and three patient primary cells to different concentrations of TIG in combination with HHT on the viability 392 of the cells was measured by an MTS assay after a $72 \mathrm{~h}$ incubation. Viability was determined by MTS staining, 393 and the results were expressed as a percentage of the results for untreated cells. ***p $<0.001$; **p $<0.01$, as 394 determined by a Tukey's test after a one-way ANOVA. Error bars represent the mean \pm SD.

Figure 3. Synergistic effect of the sequential combination of agents in inhibiting myeloid leukemia cell proliferation. A. Colony formation of the myeloid leukemia cell lines, SKM-1, MDS-L, and MOLM-13 cells were treated with TIG, HHT, or TIG combined with HHT, respectively. The survival of myeloid leukemia cells was evaluated by colony formation assays using Image $\mathbf{J}$ software. B. Apoptosis induced by various treatments at $72 \mathrm{~h}(* * \mathrm{p}<0.01$, one-way ANOVA, combination treatment versus control and single treatment). C. The survival of myeloid leukemia cells was evaluated using a colony formation assay. Mean \pm SD. ***p $<0.001 ; *$ * $<0.01 ; * \mathrm{p}<0.05$. D. Western blot of cleaved Caspase-3 and cleaved PARP-1 in myeloid leukemia cells. $\beta$-actin served as a loading control.

Figure 4. The combination of TIG and HHT has in vivo anti-leukemia activity. A. Differences in the tumor volume between combination treatments versus control and single treatments are shown after two weeks after drug treatment. B and C. The tumor mass and the mean volume display significant statistical differences in the tumor volume between the combination therapy and the control and single therapy. $* * p<0.001$ as determined by a Tukey's test after a one-way ANOVA. Error bars represent the mean \pm SD. D. The nude mouse weight during the course of drug treatment. Error bars represent the mean \pm SD. E. Immunohistochemical detection of the expression of cell proliferation-related molecules in the tumor tissue. 
410 Figure 5. TIG exhibits antileukemia activity with HHT by downregulating mTOR/4EBP1 pathway

411 proteins and mitochondrial gene expression. A. Western blot of cleaved Caspase-3, cleaved PARP-1, and

$412 \beta$-actin in the tumor tissue. B. Effects of TIG and HHT on mitochondrial translation protein levels of Cox-1,

413 Cox-4, and $\beta$-actin. C. Effects of TIG and HHT treatment alone or in combination on the levels of

414 mitochondrial translation proteins, Cox-1, Cox-4, and $\beta$-actin. D. Effects of TIG and HHT on the levels of p-

415 Ractor, p-AKT, p-mTOR, p-Raptor, p-4EBP1, and $\beta$-actin in SKM-1,MDS-L, and MOLM-13 protein

416 expression and three primary cells treated for $72 \mathrm{~h}$. $\beta$-actin was used as a loading control. 


\section{Figures}

$24 \mathrm{H}$
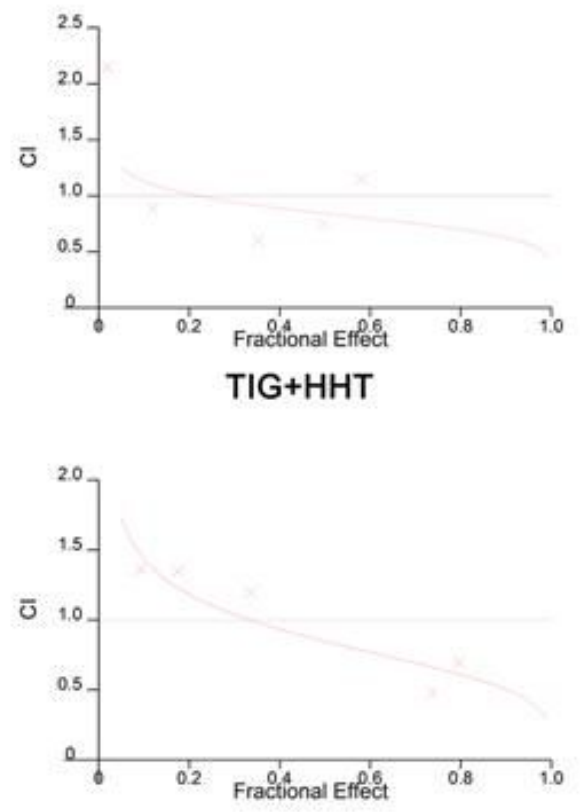

$\mathrm{TIG}+\mathrm{CHI}$

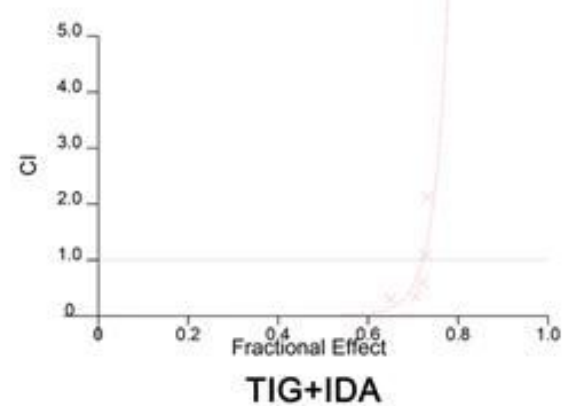

$48 \mathrm{H}$
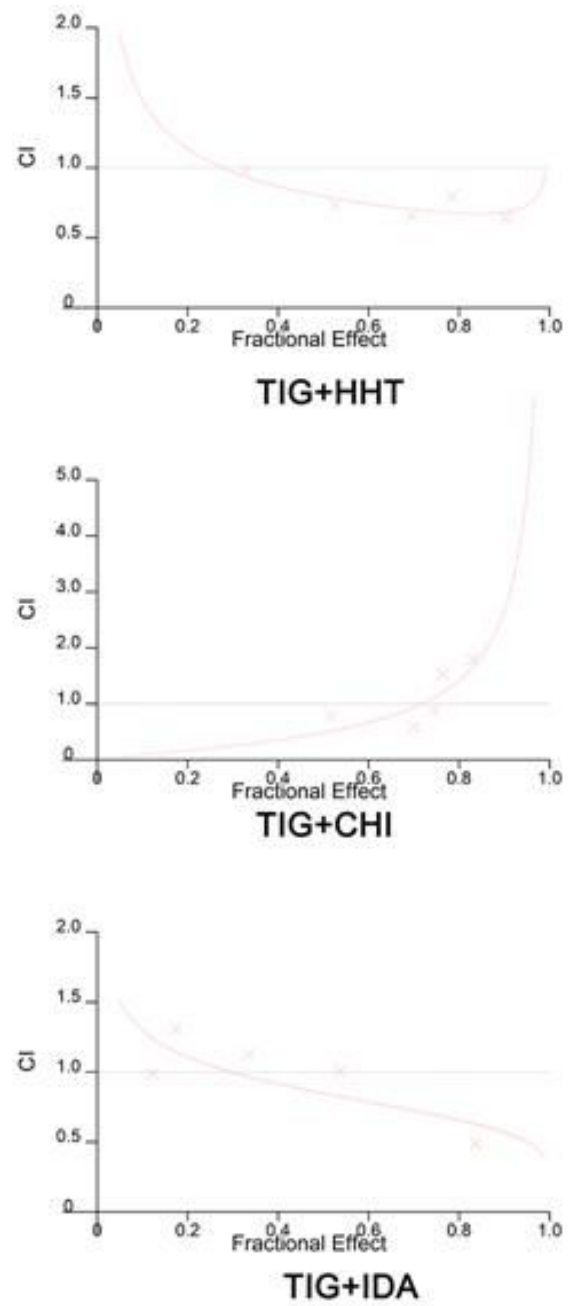

$72 \mathrm{H}$
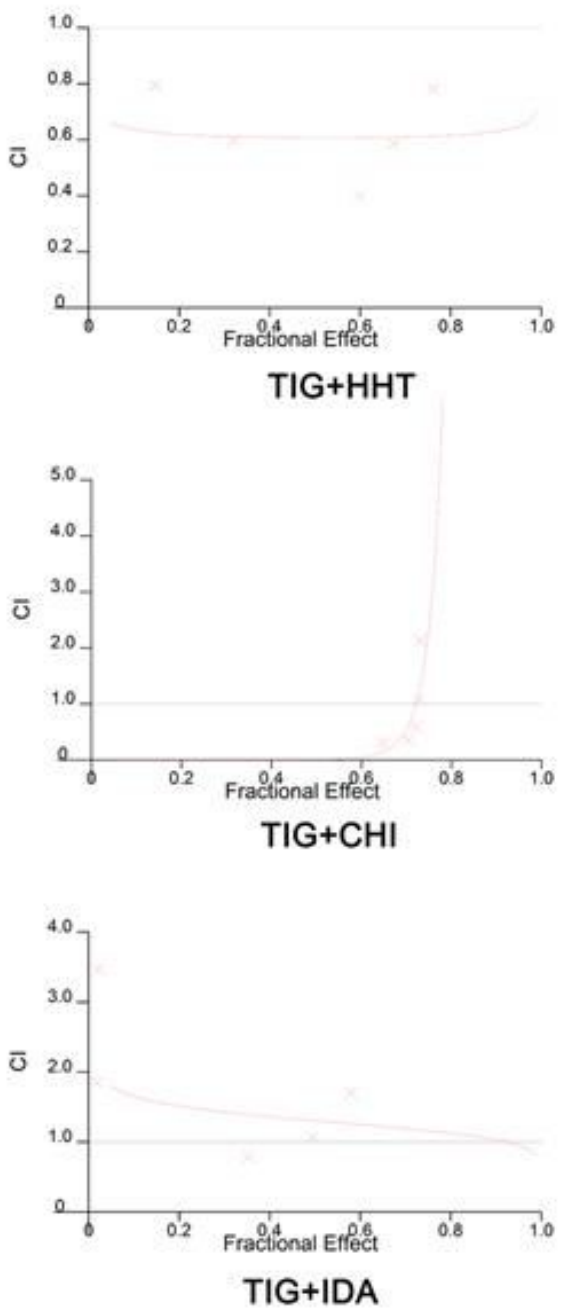

\section{Figure 1}

TIG has in vitro antileukemia activity in combination with HHT. The combination index (Cl) of TIG used in combination with IDA, HHT, or CHI in the myeloid leukemia cell line, SKM-1, was determined by MTS staining after a three day drug culture and was calculated using CalcuSyn software (three experiments each). The effect of a $72 \mathrm{~h}$ exposure of SKM-1 cells on different concentrations of TIG in 381 combination with IDA, HHT, and CHI on cell viability was measured using an MTS assay after a $72 \mathrm{~h}$ incubation. The data were analyzed using Calcusyn software to generate a $\mathrm{Cl}$ versus fractional effect (cell death) plot showing the effect of a combination of TIG with IDA, $\mathrm{HHT}$, or $\mathrm{CHI}$. $\mathrm{Cl}<1$ indicates synergism. 

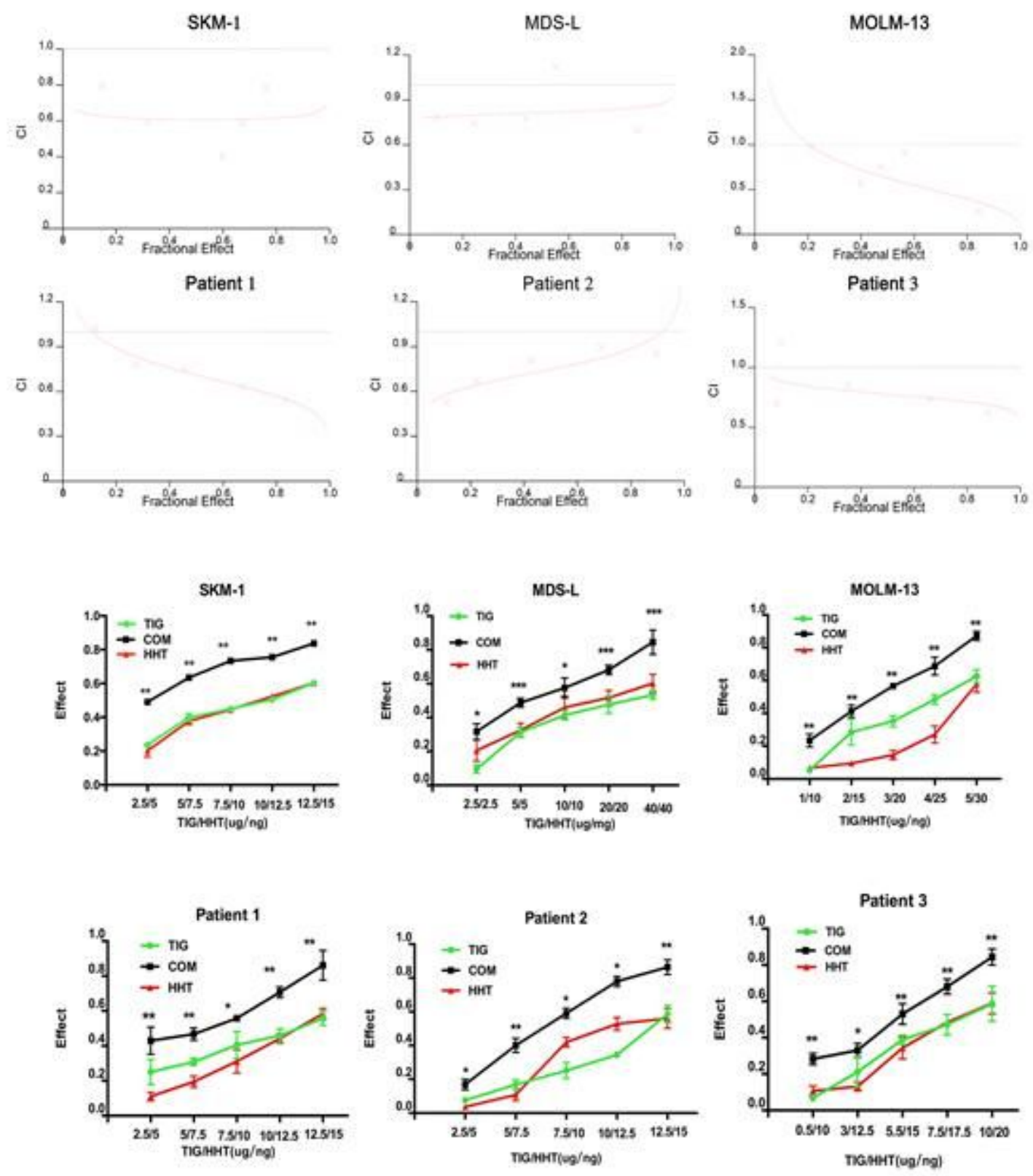

\section{Figure 2}

Combination treatment using compounds targeting leukemic cells and patient primary cells identifies a synergistic effect. A. Cl plots of DAC and IDA sequential combination in human leukemia cell lines. Cells were treated as previously described. The combinations were used at fixed molar ratios based on 387 the IC50 values of each drug. The effects of the combinations were estimated using the CalcuSyn software, which was developed based on the median-effect method. $\mathrm{Cl}<0.8$ indicates synergy; $\mathrm{Cl}=0.8$ to 1.2 is additive; 18 and $\mathrm{Cl}>1.2$ indicates antagonism. $\mathrm{B}$. The effect of a $72 \mathrm{~h}$ exposure on SKM-1, MDS-L, and MOLM-13 cells and three patient primary cells to different concentrations of TIG in combination with HHT on the viability of the cells was measured by an MTS assay after a $72 \mathrm{~h}$ incubation. Viability was determined by MTS staining, and the results were expressed as a percentage of the results for untreated 
cells. ${ }^{* \star} \mathrm{p}<0.001 ;{ }^{*} \mathrm{p}<0.01$, as determined by a Tukey's test after a one-way ANOVA. Error bars represent the mean $\pm \mathrm{SD}$.

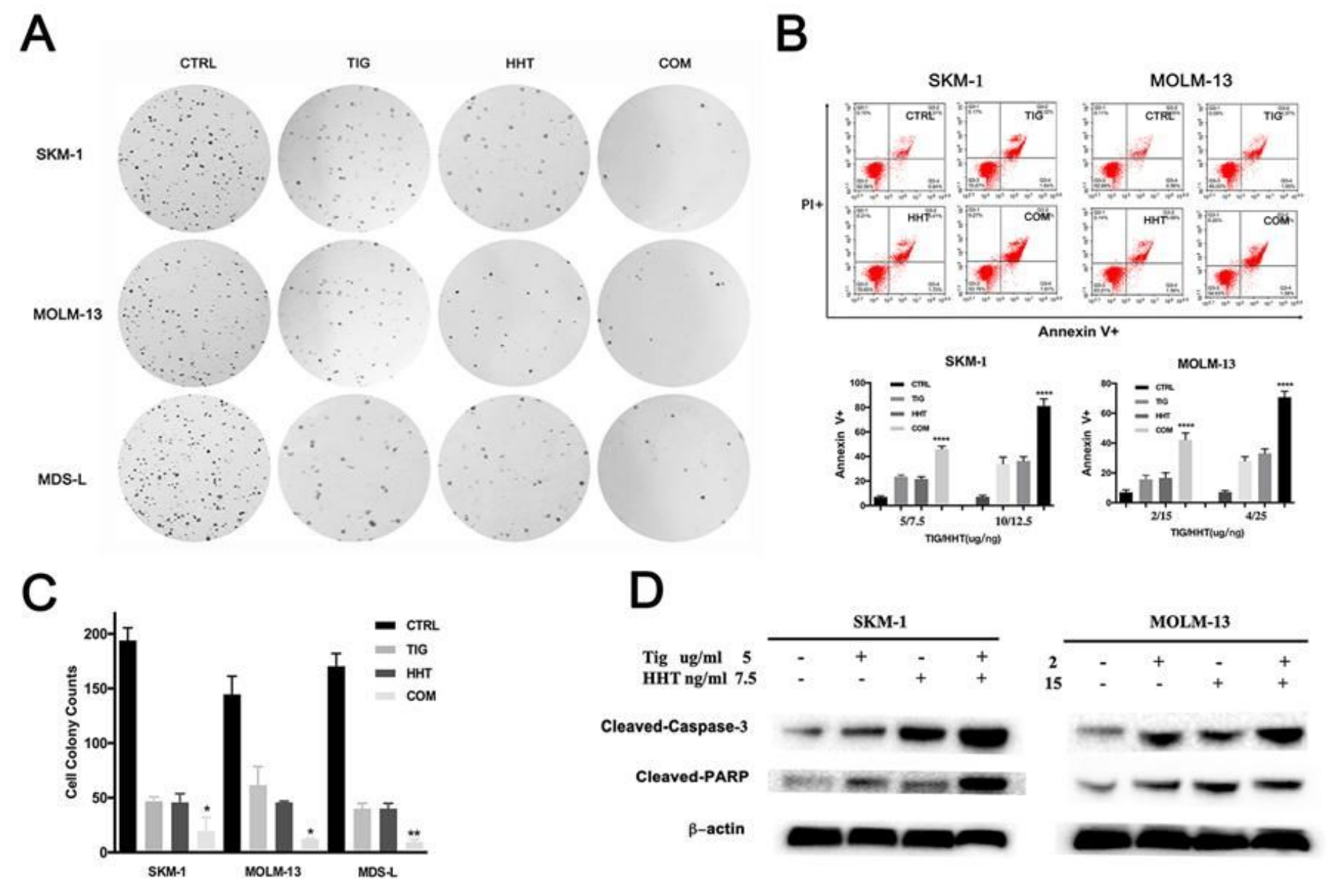

\section{Figure 3}

Synergistic effect of the sequential combination of agents in inhibiting myeloid leukemia cell proliferation. A. Colony formation of the myeloid leukemia cell lines, SKM-1, MDS-L, and MOLM-13 cells were treated with TIG, HHT, or TIG combined with HHT, respectively. The survival of myeloid leukemia cells was evaluated by colony formation assays using Image $\mathrm{J}$ software. B. Apoptosis induced by various treatments at $72 \mathrm{~h}\left({ }^{* *} \mathrm{p}<0.01\right.$, one-way ANOVA, combination treatment versus control and single treatment). C $\otimes T$ The survival of myeloid leukemia cells was evaluated using a colony formation assay. Mean \pm SD. ${ }^{* \star *} p<0.001 ;{ }^{* *} p<0.01 ;{ }^{*} p<0.05$. D凶Western blot of cleaved Caspase-3 and cleaved PARP-1

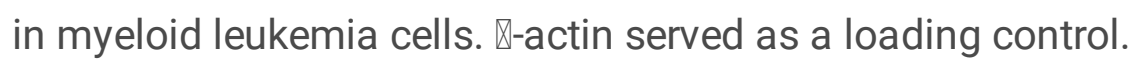




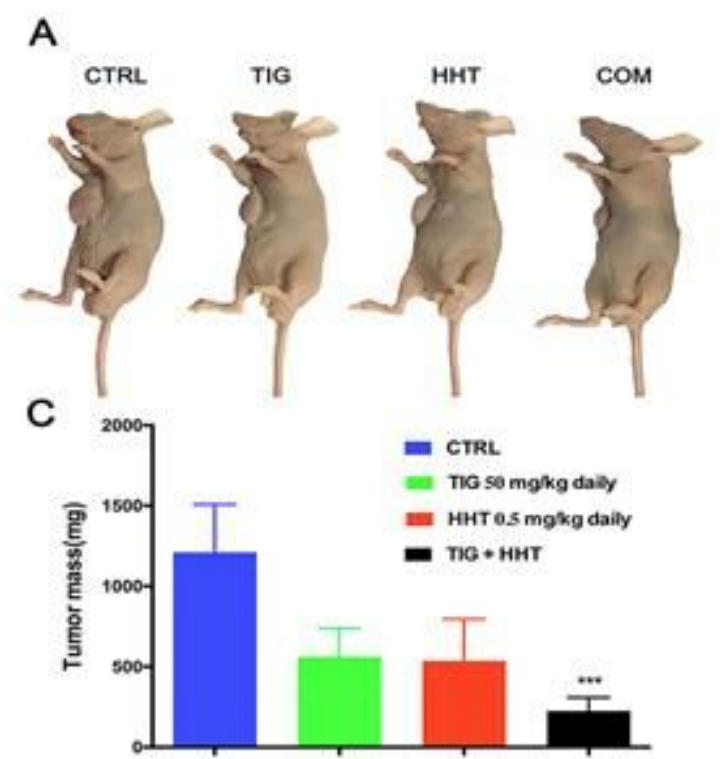

B

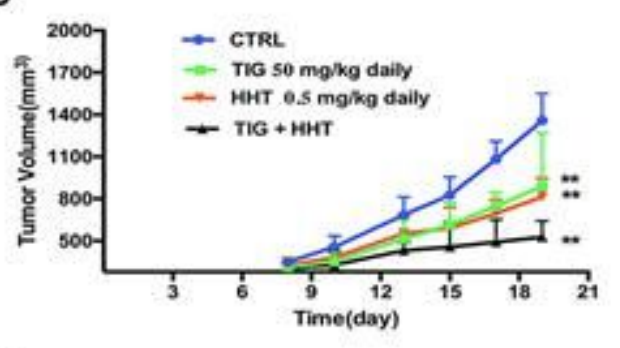

D

E

Tis
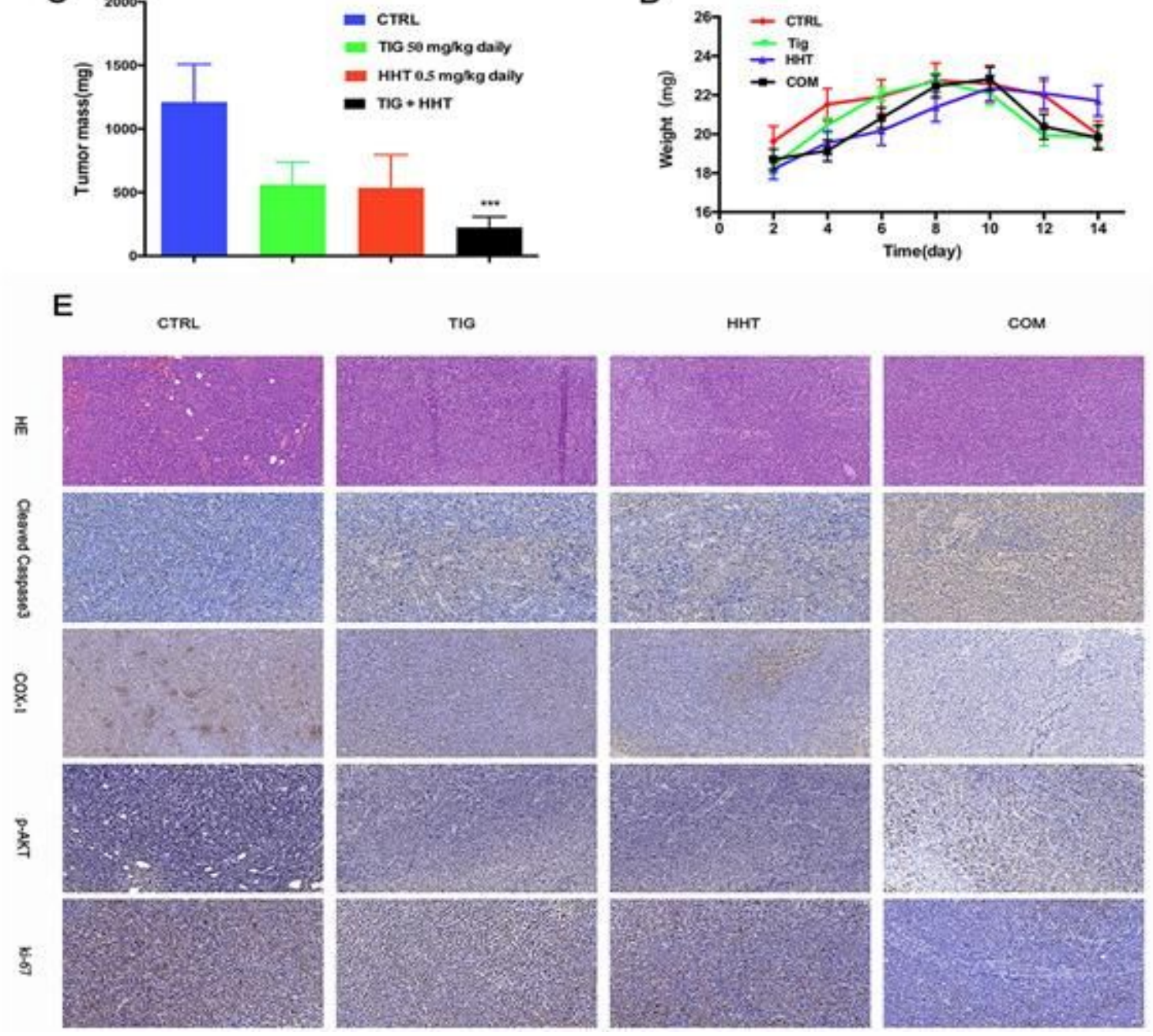

\section{Figure 4}

The combination of TIG and HHT has in vivo anti-leukemia activity. A. Differences in the tumor volume between combination treatments versus control and single treatments are shown after two weeks after drug treatment. B and $\mathrm{C}$. The tumor mass and the mean volume display significant statistical differences in the tumor volume between the combination therapy and the control and single therapy. ${ }^{* \star} p<0.001$ as determined by a Tukey's test after a one-way ANOVA. Error bars represent the mean \pm SD. D. The nude mouse weight during the course of drug treatment. Error bars represent the mean $\pm S D$. $E$. Immunohistochemical detection of the expression of cell proliferation-related molecules in the tumor tissue. 
A

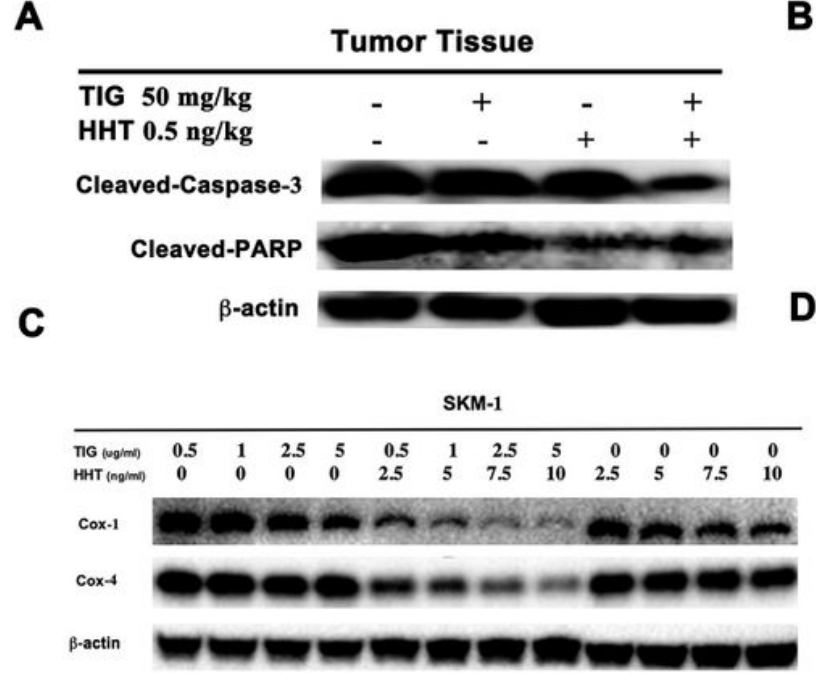

B
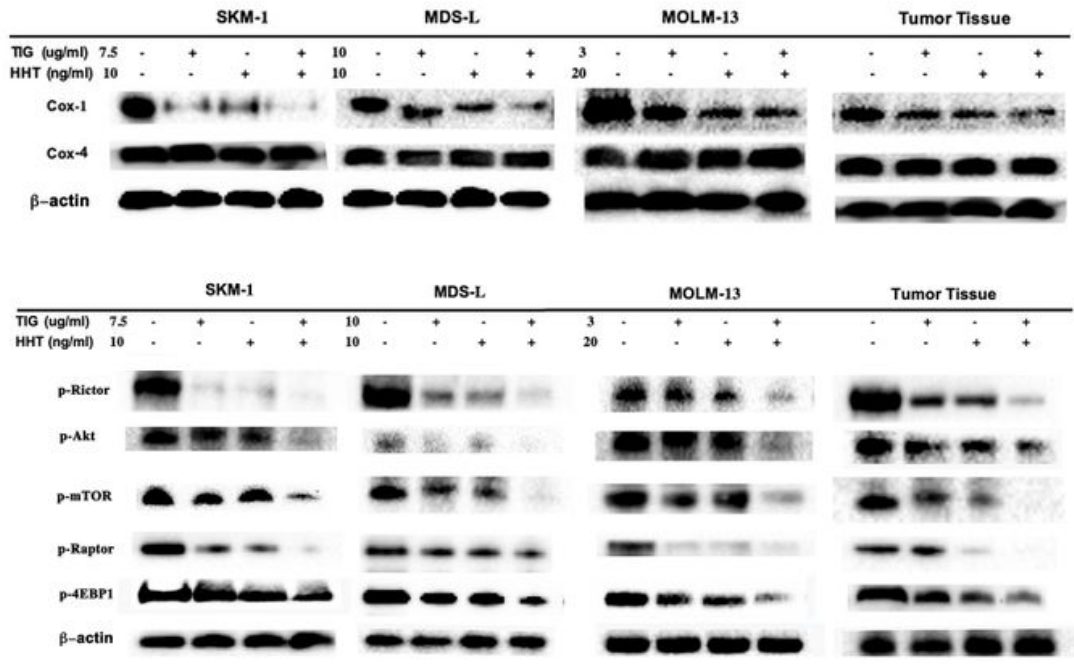

\section{Figure 5}

TIG exhibits antileukemia activity with HHT by downregulating mTOR/4EBP1 pathway proteins and

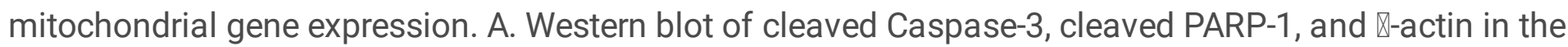

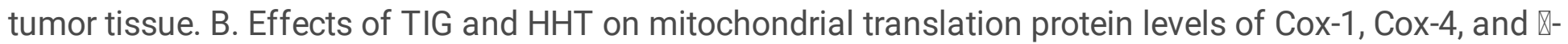
actin. C. Effects of TIG and HHT treatment alone or in combination on the levels of mitochondrial translation proteins, Cox-1, Cox-4, and $\rrbracket$-actin. D. Effects of TIG and HHT on the levels of p- Ractor, p-AKT,

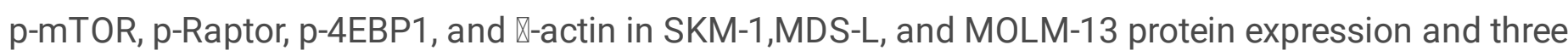
primary cells treated for $72 \mathrm{~h}$. $\mathbb{B}$-actin was used as a loading control.

\section{Supplementary Files}

This is a list of supplementary files associated with this preprint. Click to download.

- Tables1.pdf

- Tables2.pdf

- Tables3.pdf

- Tables4.pdf 